\title{
Molecular detection and phylogenetic analysis of West Nile virus lineage 2 in sedentary wild birds (Eurasian magpie), Greece, 2010
}

G Valiakos ${ }^{1,2}$, A Touloudi, ${ }^{1,2}$, C lacovakis ${ }^{1,2}$, L Athanasiou, ${ }^{1,2}$, P Birtsas 3,4, V Spyrou ${ }^{5}$, C Billinis (billinis@vet.uth.gr)

1. Department of Microbiology and Parasitology, Faculty of Veterinary Medicine, University of Thessaly, Karditsa, Greece

2. Laboratory of Zoonoses Research, Institute of Biomedical Research and Technology (BIOMED/CERETETH), Larissa, Greece

3. Department of Forestry and Natural Environment Administration, Technological Education Institute of Larissa, Karditsa, Greece

4. Hunting Federation of Macedonia and Thrace, Thessaloniki, Greece

5. Department of Animal Production, Technological Education Institute of Larissa, Larissa, Greece

Citation style for this article:

Valiakos G, Touloudi A, Iacovakis C, Athanasiou L, Birtsas P, Spyrou V, Billinis C. Molecular detection and phylogenetic analysis of West Nile virus lineage 2 in sedentary wild birds (Eurasian magpie), Greece, 2010.

Euro Surveill. 2011;16(18):pii=19862. Available online: http://www.eurosurveillance.org/ViewArticle.aspx?Articleld=19862

A West Nile virus (WNV) lineage 2 strain was molecularly identified and characterised in a Eurasian magpie hunted in Greece in 2010, during a WNV outbreak in humans. Phylogenetic analysis revealed the highest sequence similarity ( $299 \%$ ) with other WNV lineage 2 strains derived from birds of prey in Austria and Hungary (2004-2009). This first molecular detection of WNV in sedentary wild birds in Greece, which are possible reservoirs of the virus, is a public health concern.

\section{Introduction}

West Nile virus (WNV) is a mosquito-transmissible Flavivirus with zoonotic potential. The virus has been present in Europe for decades; however, only recently were strains of lineage 2 (L2) identified outside of Africa: in 2004 and 2005 in goshawks in Hungary, in 2007 in Volgograd, Russia, and in 2008 and 2009 in goshawks and a falcon in Austria [1-3]. From early July through October 2010, 261 laboratory-confirmed cases of WNV infection in humans were reported in northern Greece as part of an outbreak. Of these, 191 patients presented with neuro-invasive symptoms, and 34 deaths were reported [4]. Most cases were observed in central Macedonia, in areas located between four major rivers (Axios, Loudias, Aliakmon and Gallikos) which converge into a common delta, a well-known resting and breeding ground for migratory birds.

\section{Methods and results}

The objective of our study was to detect possible infection of wild birds with WNV during the outbreak in Greece, and to molecularly characterise and define the WNV strain geographical origin in positive samples.

Our first focus was on members of the Corvidae family. Many corvid species are sedentary and territorial, having a wide daily dispersal range of up to $20 \mathrm{~km}$, social, roosting in large colonies and abundant in both wetlands and urban areas [5]. Hence, introduction of the virus in an area (i.e. via migratory birds) may result in its transmission, circulation and maintenance in local corvid populations. Samples from hunterharvested corvids (Eurasian magpies and carrion crows, hunted species according to Greek law) were collected during the hunting season (from 20 August until 28 February the following year) of 2009/10 and of 2010/11. Sampling was carried out in the municipalities of Thermi and Axios (prefecture of Thessaloniki, central Macedonia, Greece) by members of the Hunting Federation of Macedonia and Thrace, locating corvid roosting sites in nearby wetlands. Hunters were briefed on signs of encephalitis in birds, and were instructed to report any such observations. No findings of birds with signs of encephalitis or dead birds were reported from any of the hunters.

Of 96 corvids collected, 36 were tested, including 28 Eurasian magpies (Pica pica) and eight carrion crows (Corvus corone). A pool of selected tissues (kidney, heart, liver) was created from each bird. RNA was extracted from each pool, which constituted a single sample, using the PureLink RNA Mini Kit (Invitrogen). An -RT-PCR specific for Japanese encephalitis virus complex was performed for all extracts resulting in a 1,084-bp amplification product covering part of the nonstructural protein 5 (NS5) gene, as described earlier [6]. A band of expected size was obtained from one PCR product derived from a magpie harvested near the village of Trilofos $\left(40^{\circ} 28^{\prime} 25.57^{\prime \prime} \mathrm{N}, 22^{\circ} 58^{\prime} 28.62\right.$ 'E) in September 2010 (Figure 1). A serum sample from the magpie in question was tested for the presence of WNV IgG antibodies by indirect immunofluorescence test using a commercial kit (EUROIMMUN) [7]; the serum sample was positive at a dilution of $1 / 30$.

The positive $P C R$ product was purified using the PureLink PCR Purification Kit (Invitrogen) and was 
bidirectionally sequenced using the fluorescent BigDye Terminator Cycle sequencing kit v3.1 (Applied Biosystems), followed by fragment separation with a 3,730xl DNA Analyzer (Applied Biosystems).

Phylogenetic analysis was conducted using MEGA 3.1 [9]. Nucleotide sequences from other WNV strains were retrieved from Genbank (NCBI). Phylogenetic analysis of 797 nucleotide-long partial NS 5 sequences was performed. A neighbour-joining phylogenetic tree using Kimura-2 parameter distance matrix was inferred from 26 WNV strain sequences (including that derived from the magpie in our study) and two sequences of the Japanese Encephalitis virus complex as outgroups (Figure 2). Node support was assessed with 1,000 bootstrap pseudo-replicates.
The WNV sequence derived from the Greek magpie clustered with WNV L2 strain sequences and presented highest (99.9\%) sequence similarity to L2 strain sequences derived from birds of prey in Austria obtained in 2008 and 2009 [2]. A 99.6\% similarity was also observed with the corresponding region of an L2 strain derived from a dead goshawk in Hungary in 2004 [1]. No amino acid changes were observed in the genomic region of the magpie derived WNV strain compared to Austrian and Hungarian strains. According to our analysis, all these strains as well as two strains from South Africa belong to the same sub-cluster. A lower sequence similarity (96.8\%) was observed with a WNV L2 strain isolated during an outbreak in Russia in 2007. The Russian strain sequence groups with other African strains (including other South African strains) in

\section{FIGURE 1}

Bird sampling area for West Nile Virus, Central Macedonia, Northern Greece, 2009-2011

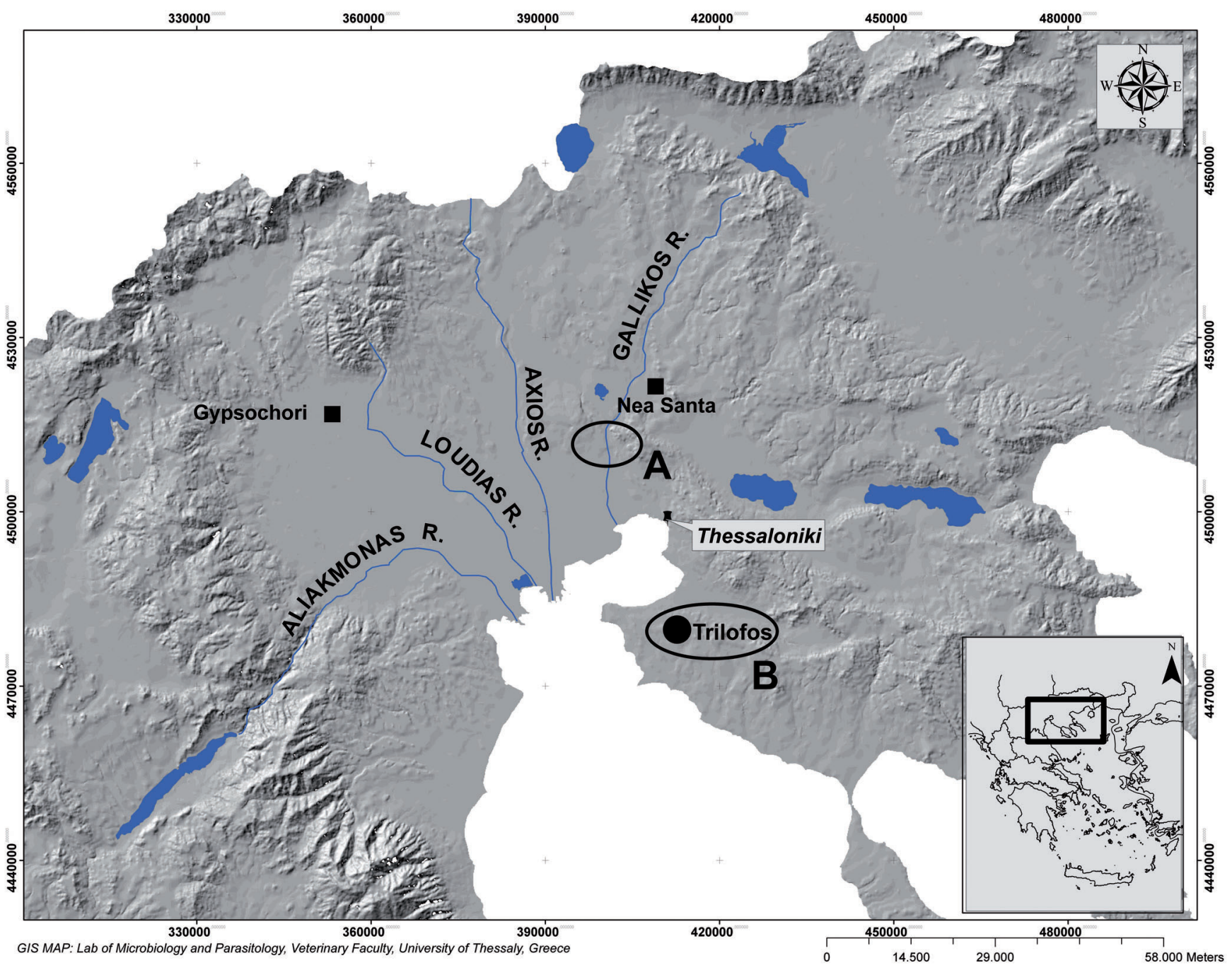

WNV: West Nile Virus.

The study area corresponds to the areas where most human cases occurred during the WNV outbreak.

Black square boxes indicate where WNV was detected in mosquitoes [8].

$A$ and $B$ indicate areas, where tested corvids were harvested.

The black circle indicates where the WNV-positive Eurasian magpie was hunted. 
a separate sub-cluster, suggesting a different reintroduction of WNV L2 in Europe [3]. The sequence from the Greek magpie isolate was deposited in GenBank under accession no. JF719073.

\section{Discussion}

From early July through October 2010, a WNV outbreak in humans occurred in northern Greece, as confirmed by serologic evidence. To date, no WNV genomic sequences are available from the human cases during this outbreak. A WNV strain sequence derived from a magpie hunted during the outbreak of the human disease was found in this study. The sequence has highest sequence similarity to L2 strain sequences from birds of prey in Austria obtained in 2008 and 2009. WNV RNA fragments, though limited in size, (146 nt $\mathrm{NS}_{5}$ genomic region) with $100 \%$ sequence similarity to Hungarian and Austrian L2 strains, were also detected in two pools of mosquitoes caught during the time of the Greek outbreak and in the same area [8]. The mosquito WNV sequence was not included in our analysis because it did not overlap with the magpie WNV sequence. However, the similarity of both to the Austrian L2 strain sequences suggests that the same WNV strain is implicated in the magpie and mosquito infections and associated with the human outbreak. The evidence may implicate this corvid species in local virus maintenance and generates concerns about possible overwintering and expansion of the virus in neighbouring areas. To test this hypothesis, research must be extended in non-epidemic periods, by performing molecular and serologic surveillance in wild birds and focusing efforts on the isolation of infectious WNV from avian samples.

Phylogenetic analysis of our strain revealed a high sequence similarity with Austrian and Hungarian WNV strains detected in previous years in birds of prey (2004-2009). According to these findings, it can be hypothesised that the virus expanded from northern Europe southwards. The area of the recent outbreak is a well-known resting and breeding ground for migratory birds passing on the way from nesting grounds in Europe to wintering areas in Africa. Re-introduction of the virus in the future by birds migrating along the south-eastern migration route that leads from Europe and western Asia to Africa should also be considered possible and needs further investigation.

\section{Acknowledgments}

The research leading to these results received partial funding from the European Union Seventh Framework Programme (2007-2013) under grant agreement no. 222633 (WildTech).

\section{FIGURE 2}

Phylogenetic tree of West Nile Virus strains based on nt sequences of the NS5 genomic region

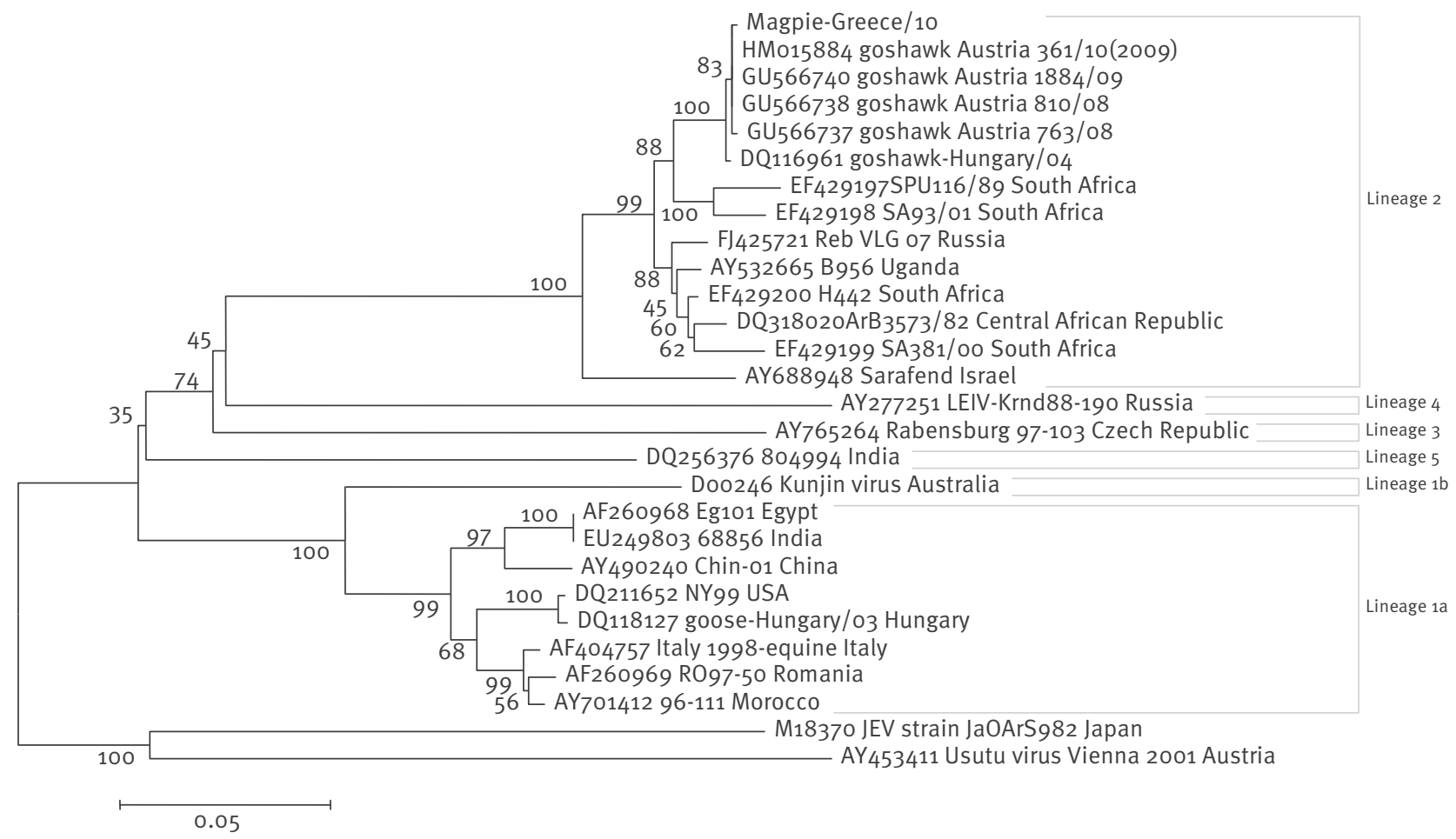

The sequence from the present study is shown in bold.

The sequences used to derive the phylogenetic tree were $797 \mathrm{nt}$ long.

GenBank accession numbers and geographic origins of strains are shown.

Bootstrap values (in per cent) are represented at each tree node. 


\section{References}

1. Bakonyi T, Ivanics E, Erdelyi K, Ursu K, Ferenczi E, Weissenbock $\mathrm{H}$, et al. Lineage 1 and 2 strains of encephalitic West Nile virus, central Europe. Emerg Infect Dis. 2006;12(4):618-23.

2. Wodak E, Richter S, Bagó Z, Revilla-Fernández S, Weissenböck $\mathrm{H}$, Nowotny $\mathrm{N}$, et al. Detection and molecular analysis of West Nile virus infections in birds of prey in the eastern part of

Austria in 2008 and 2009. Vet Microbiol. 2011;149(3-4):358-66.

3. Platonov AE, Fedorova MV, Karan LS, Shopenskaya TA

Platonova OV, Zhuravlev VI. Epidemiology of West Nile

infection in Volgograd, Russia, in relation to climate change and mosquito (Diptera: Culicidae) bionomics. Parasitol Res. 2008;103 Suppl 1:S45-53.

4. Hellenic Centre for Disease Control and Prevention (KEELPNO). West Nile Virus Epidemic in Greece. Available from:

5. http://www.keelpno.gr/index.php?option=com co ntent \&view $=$ article\&id $=140 \% 3$ Awest-nile-virusinfection\&catid=45\%3A2010-06-28-08-41-59\&ltemid =1

6. Reiter P. West Nile virus in Europe: understanding the present to gauge the future. Euro Surveill. 2010;15(10): pii=19508. Available from: http://www.eurosurveillance.org/ViewArticle. aspx?Articleld $=19508$

7. Weissenböck H, Kolodziejek J, Url A, Lussy H, Rebel-Bauder B, Nowotny N. Emergence of Usutu virus, an African mosquitoborne flavivirus of the Japanese encephalitis virus group, central Europe. Emerg Infect Dis. 2002;8(7):652-6.

8. Ziegler U, Seidowski D, Globig A, Fereidouni SR, Ulrich RG, Groschup MH. Sentinel birds in wild-bird resting sites as potential indicators for West Nile virus infections in Germany. Arch Virol. 2010;155(6):965-9.

9. Papa A, Xanthopoulou K, Gewehr S, Mourelatos S. Detection of West Nile virus lineage 2 in mosquitoes during a human outbreak in Greece. Clin Microbiol Infect. 2011; 17: no. doi: 10.1111/j.1469-0691.2010.03438.x

10. Kumar S, Tamura K, Nei M. MEGA3: Integrated software for Molecular Evolutionary Genetics Analysis and sequence alignment. Brief Bioinform. 2004;5(2):150-63. 